\title{
Comparison of the accuracy of hysteroscopy and hysterosalpingography in evaluation of the uterine cavity in patients with recurrent pregnancy loss
}

Received: 29 December 2004 / Accepted: 6 April 2005 / Published online: 2 June 2005

(c) Springer-Verlag Berlin / Heidelberg 2005

\begin{abstract}
The aim of this study was to compare the accuracy of hysteroscopy and hysterosalpingography (HSG) in evaluating the uterine cavity in patients with recurrent pregnancy loss (RPL). One hundred and twenty consecutive patients with a history of RPL were enrolled into this prospective-observational study in the reproductive endocrinology and infertility clinic of a tertiary referral center. Diagnostic office hysteroscopy without anesthesia or sedation, HSG, and diagnostic laparoscopy when indicated were performed in each case. Eighty-five of 120 (70.83\%) hysteroscopic studies performed for RPL demonstrated an acquired (55 cases: $64.7 \%$ ) or congenital (30 cases: $35.3 \%$ ) intrauterine lesion. Furthermore, several other etiologic factors were also identified in RPL patients with intrauterine lesions. HSG accurately diagnosed an intrauterine defect in only 56 of $85(65.88 \%)$ cases, based on hysteroscopic confirmation. Fifty percent of the cases with incomplete uterine septum were overlooked during HSG. The sensitivity, specificity, positive predictive value, and negative predictive value of HSG were $74.6 \%, 79.5 \%$, $90.4 \%$, and $54.7 \%$, respectively. There was a single complication $(0.83 \%)$ due to hysteroscopy. Hysteroscopy is more accurate than HSG in evaluating the uterine cavity in patients with RPL. We recommend it as a routine procedure instead of HSG.
\end{abstract}

Keywords Recurrent pregnancy loss - Uterine cavity evaluation · Hysteroscopy · Hysterosalpingography

H. Camuzcuoglu $\cdot$ Y. Yildirim $(\bowtie) \cdot$ S. Sadik

S. Kurt $\cdot$ S. Tinar

Department of Reproductive Endocrinology and Infertility,

SSK (Social Security Agency) Aegean Obstetrics

and Gynecology Teaching Hospital, 2933 Sok,

Palmiye Sit, Bahar Mar,

35380 Izmir, Turkey

E-mail: ykuzeyli@mynet.com

\section{Introduction}

Recurrent pregnancy loss (RPL) is traditionally defined as three or more consecutive pregnancy losses [1]. Despite this classic definition, detailed work-up is often delayed until the third miscarriage [2]. RPL is estimated to occur with $2-4 \%$ of reproductive-age couples, and the chance for a subsequent abortion increases with each successive abortion [3]. These women, however, still have a $60-70 \%$ chance of delivering a subsequent pregnancy [4-6], and approximately $82 \%$ of them have a live birth $(78 \%$ of primary aborters and $86 \%$ of secondary aborters) [7].

Previous data indicated no difference in the prevalence of etiologies of RPL in couples with two or more abortions compared with those with three or more [8], and if a full evaluation is completed on couples with either two or three consecutive losses, the etiology of RPL remains unknown in approximately one-half to two-thirds of all cases [4, 5]. However, it is now widely accepted that RPL is a heterogeneous condition with several etiological factors such as anatomic (congenital müllerian anomalies, Asherman's syndrome, leiomyomata, polyps), genetic (balanced translocation in the parents, chromosomal aberrations in the fetuses, factor Leiden mutation, hyperhomocysteinemia), endocrine (luteal phase deficiency, hyperprolactinemia, hypothyroidism, insulin resistance with or without polycystic ovarian syndrome, uncontrolled diabetes mellitus), infectious (mycoplasma, ureaplasma, chlamydia), autoimmune and immunologic (elevated levels of anticardiolipin or antiphospholipid antibodies, lupus anticoagulant), and environmental factors. Psychological factors [9] and familial predisposition [10] have been claimed as well. Currently, human leukocyte antigen (HLA)-G polymorphism [11], elevated plasma tumor necrosis factor (TNF)-alpha levels [12], and abnormal expression of CD56 + cells by the endometrium [13] have also been related to recurrent spontaneous abortions. Generally, more than one etiologic factor is 
present in the same case. Congenital and acquired uterine abnormalities, which are potentially correctable factors, consist of a majority of the explained cases of the RPL, with rates of 15-38\% [14-19]. Because of the high prevalence of uterine abnormalities, evaluation of the uterine cavity should be routinely done in the basic assessment of women with RPL.

Historically, dilatation and curettage, hysterosalpingography (HSG), transvaginal sonography, office hysteroscopy, hysterosonography, and magnetic resonance imaging have been applied to evaluate the uterine cavity. Hysteroscopy, however, is still widely used because it has high accuracy rates up to $98 \%$ [20, 21]. Since the first hysteroscopic intervention was reported in 1869 by Pantaleoni [22], and parallel with advances in instrumentation and operative technique in the last 35 years, hysteroscopy has became a frequent procedure in gynecology practice, especially for evaluating cases of reproductive failure [23-25]. Currently, the diagnostic and therapeutic applications of hysteroscopy are many and range from visualizing endometrial malignancy to examining the fetus in utero.

The aim of this study was to compare the accuracy of hysteroscopy and HSG and to evaluate the impact of hysteroscopy as a routine procedure in evaluating the uterine cavity in patients with RPL.

\section{Materials and methods}

Between April 2001 and August 2003, a total of 121 consecutive women with a history of RPL were prospectively recruited from the SSK (Social Security Agency) Aegean Obstetrics and Gynecology Teaching Hospital, Department of Reproductive Endocrinology and Infertility, a tertiary referral center for patients with RPL in Izmir, Turkey. This prospective observational study was approved by the institutional review board, and informed consent was obtained from each patient after the purpose and nature of the study had been fully explained.

Pregnancy loss was defined as any natural abortion occurring before 24 weeks of gestation or with a fetal weight of less than $500 \mathrm{~g}$. RPL was defined as two or more consecutive, spontaneous pregnancy losses whether or not the patient had a previous or subsequent successful pregnancy outcome.

Exclusion criteria were an ongoing or suspected pregnancy or a history of iodine allergy or reactions. Patients with signs of pelvic inflammatory disease and those undergoing active treatment for any sexually transmitted disease were also not enrolled into the study.

In all cases before hysteroscopy, HSG using an aqueous contrast medium (Omnipaque; Opakim, Turkey) was performed as part of the investigations for uterine abnormalities. All HSGs and hysteroscopies were performed during the follicular phase. Sensitivity, specificity, positive predictive value (PPV), and negative predictive value (NPV) were calculated for HSG, considering hysteroscopic diagnosis as the gold standard.
Hysteroscopy was carried out with a rigid office hysteroscope and a diagnostic sheath with a $5-\mathrm{mm}$ diameter (Endomat, Karl Storz Endoscopy, Tuttlingen, Germany). It was carried out as an outpatient procedure. To avoid discomfort or pain, cervical dilatation was not performed in any patient. Prophylactic oral doxycycline (daily 100-mg tablets for 5 days) was used in any subject who had a prior history of pelvic inflammatory disease. All study patients took naproxen sodium (500-mg oral tablets) $30 \mathrm{~min}$ before each outpatient procedure. Except in five cases, no local or general anesthesia or sedation were used in the patients. Distension of the uterine cavity was obtained using glycine solution $(400 \mathrm{ml} / \mathrm{min})$, and the intrauterine pressure, ranging from $175 \mathrm{~cm}$ to $275 \mathrm{~cm} \mathrm{H}_{2} \mathrm{O}$, was automatically controlled by an electronic irrigation and suction device (Endomat, Karl Storz, Tuttlingen, Germany). A study was judged adequate only when the entire uterine cavity and both tubal ostia were visualized during the procedure.

Uterine abnormalities detected during endoscopic examinations were divided into two categories: congenital or acquired pathologies. Congenital uterine defects were classified in accordance with the modified American Fertility Society classification [26]. Patients with hysteroscopic evidence of incomplete or complete uterine septum underwent laparoscopic confirmation to evaluate the uterine fundal contour. The diagnosis of a septum was made if the external fundal contour was convex, flat, or smooth and minimally depressed in the midline less than $1 \mathrm{~cm}$ from the fundal contour. A bicornuate uterus was defined as one with a fundal indentation greater than $1 \mathrm{~cm}$ or with a cleft of any size that sharply demarcated the midline [27]. Submucosal fibroids and endometrial polyps detected during hysteroscopy were removed by resectoscope.

Regardless of the results of hysteroscopy, all women with RPL were also screened for possible concomitant causes of miscarriage: peripheral blood karyotype of both parents; tests of insulin resistance (diagnosed as fasting insulin levels greater than $20 \mu \mathrm{U} /$ $\mathrm{ml}$ or a fasting-glucose-to-fasting-insulin ratio of less than 4.5); thyroid function, serum prolactin, and midluteal plasma progesterone (normal, greater than $20 \mathrm{nmol} / \mathrm{l})$ measurements; measurements of lupus anticoagulant (dilute Russell viper venom test), anticardiolipin antibodies (ACA), antinuclear antibodies (ANA), and antithyroid antibodies; cervical cultures for mycoplasma, ureaplasma, and chlamydia; and toxoplasmosis serology.

\section{Results}

A total of 121 patients were investigated with hysteroscopy; one case was excluded from the study because of failure in the insertion of the hysteroscope. Thus, 120 women who had a history of RPL were enrolled into the final study. Their mean age was 28.3 (range 23-41) years. 
These patients had had between two and eight prior pregnancy losses, with a mean of 3.62 losses. While 96 patients had had only 1st-trimester losses, 17 cases had had both 1st- and 2nd-trimester losses, and seven cases had had only 2 nd-trimester losses.

Eighty-five of $120(70.83 \%)$ hysteroscopic studies performed for RPL demonstrated intrauterine lesions. The abnormalities found during hysteroscopy were given the following diagnoses: $55(64.7 \%)$ had acquired pathologies (endometrial adhesion in 31, submucous fibroid in 19, and endometrial polyp in five), and $30(35.3 \%)$ had congenital pathologies (complete uterine septum in 16, incomplete uterine septum in eight, bicornuate uterus in five, and didelphic uterus in one). All 24 patients with hysteroscopic evidence of incomplete or complete uterine septum underwent laparoscopic confirmation to evaluate the uterine fundal contour.

Fifty-six of the patients $(46.66 \%)$ had an intrauterine abnormality that could be detected with HSG. Most of these were uterine septum filling defect or uterine wall irregularity. A summary of the hysteroscopy and HSG findings of 85 cases with intrauterine abnormalities are presented in Table 1. The sensitivity, specificity, PPV, and NPV of HSG were $74.6 \%$, $79.5 \%, 90.4 \%$, and $54.7 \%$, respectively, when hysteroscopy was accepted as the gold standard. In summary, HSG accurately diagnosed an intrauterine defect in only 56 of $85(65.88 \%)$ cases based on hysteroscopic confirmation. Although all cases of complete uterine septum were diagnosed accurately with HSG, four $(50 \%)$ of eight cases of incomplete uterine septum were overlooked during HSG.

Additionally, several possible etiologic factors apart from uterine factors were identified in RPL patients with intrauterine lesions: luteal phase deficiency in 10 $(11.76 \%)$, insulin resistance in seven $(8.24 \%)$, antiphospholipid antibodies in six (7.06\%), and chromosomal translocation in the parents in two $(2.35 \%)$.

The mean duration of the procedure was 9.82 (range $8-21)$ min. There was a single complication $(0.83 \%)$ after hysteroscopy in which a patient was hospitalized for excessive uterine bleeding.

Table 1 Hysteroscopy and hysterosalpingography (HSG) findings of patients with intrauterine pathology

\begin{tabular}{ll}
\hline Hysteroscopy & HSG findings \\
findings $N(\%)$ & $N(\%)$ \\
\hline Intrauterine adhesion & Uterine wall/cavity \\
31 $(25.83)$ & irregularity 13 (15.29) \\
Polyp or submucosal fibroid & Filling defect \\
24 (20.00) & $19(22.35)$ \\
Incomplete or complete & Incomplete or complete \\
septum 24 (20.00) & septum 20 (23.53) \\
Bicornuate uterus 5 (4.17) & Bicornuate uterus 3 (3.53) \\
Didelphic uterus 1 $(0.08)$ & Didelphic uterus 1 $(1.18)$ \\
Normal cavity & Normal cavity 29 $(34.11)$ \\
\hline
\end{tabular}

\section{Discussion}

Since the impact of uterine factors on recurrent miscarriage was emphasized by Halbrecht [28], a substantial number of studies have been published each year. Even though both congenital and acquired anatomic uterine defects can potentially affect reproductive capability, an association between müllerian anomalies and reproductive failures is significantly more obvious than with acquired lesions (e.g., intrauterine adhesion and submucosal leiomyoma) [29].

It is reported that in women with RPL who have undergone HSG or hysteroscopic examination of the uterus, müllerian anomalies have been found in approximately $10 \%$, and the reproductive history of most women with a müllerian anomaly is poor [30]. In contrast, we found that approximately one-third of the RPL cases with an intrauterine defect that could be detected by hysteroscopy had a congenital müllerian anomaly, predominantly uterine septum. The most commonly reported müllerian anomaly associated with RPL is septate uterus, for which the pretreatment reproductive wastage rate is $95 \%$. However, among these patients who receive adequate therapy, the gestational outcome has markedly improved, and $87 \%$ of the pregnancies have resulted in living infants or have progressed beyond 20 weeks of gestation [31]. It is believed that poor vascularization of the uterine septum is a cause of spontaneous abortion [32]; however, a study revealed that the vascular density in uterine septa removed at the time of metroplasty was similar to that of the normal uterine wall [33].

In our series the most common acquired lesion in cases of RPL was intrauterine adhesion. Our study also revealed that there are no certain criteria for diagnosing intrauterine adhesions with HSG and that hysterosalpingographic diagnosis of them is therefore very difficult. Intrauterine adhesions following overzealous curettage of the uterus in the postpartum period, intrauterine surgery (e.g., myomectomy), or endometritis may cause menstrual irregularity, infertility, and spontaneous abortion. Dense, avascular adhesions may interfere with implantation or placentation. However, an association between intrauterine adhesion and RPL is controversial. After hysteroscopic treatment of intrauterine adhesions, normal menstruation is restored in approximately $88 \%$ of patients who have any menstrual irregularity, including amenorrhea, hypomenorrhea, and dysmenorrheal [34]. Therefore, hysteroscopic evaluation of patients with RPL is very useful with respect both to its diagnostic and its therapeutic effects.

Hysteroscopy and HSG approach the uterine cavity in different ways, and each has different advantages and limitations. Hysteroscopy performed in an outpatient setting without general or local anesthesia in more than $90 \%$ of women [35], however, allows direct visualization of the uterine cavity and thus allows biopsy and removal of any abnormal endometrial lesions simultaneously, 
while HSG is necessary for a thorough assessment of uterine malformations, complex intrauterine adhesions, and uterine scars [36]. A study investigating the accuracy of hysteroscopy in 1,500 women revealed that hysteroscopy had sensitivity, specificity, PPV, and NPV of $94.2 \%$, $88.8 \%, 83.1 \%$, and $96.3 \%$, respectively, in predicting normal or abnormal histopathology of endometrium. This study has suggested that hysteroscopy is therefore accurate in distinguishing between normal and abnormal endometrium [37]. On the other hand, Raziel et al. [38] reported that HSG shows high false-positive $(38 \%)$ and false-negative $(28 \%)$ rates in the uterine adhesion group. We detected low sensitivity and specificity of HSG in diagnosing intrauterine defects as well. Recently, Lindheim and Morales [39] have suggested that intraoperative sonohysterography (SHG) performed concomitantly with hysteroscopy is beneficial for confirming the depth of pathology and false-negative hysteroscopy findings because of SHG's three-dimensional images. However, SHG could not be performed in any patients in our series.

An accepted rate for all complications (mechanical, media-related, infectious, and bleeding) during diagnostic hysteroscopy is less than $4 \%$. Uterine perforation or cervical trauma is the most common complication, with rates ranging between $0.7 \%$ and $2.7 \%$ [40, 41], and most cervical trauma and uterine perforations occur during dilation of the cervix [42]. Even though hysteroscopy is an operative intervention instead of a diagnostic procedure, the complication risk is also less, with a frequency of $4.8 \%$ for uterine perforation, $0.6 \%$ for uterine hemorrhage, and approximately $5.5 \%$ for metabolic abnormalities (most of which are asymptomatic,) according to Castaing et al. [43]. Their study investigating complications of operative hysteroscopy also showed that the perforation rate was greater in patients treated for synechia than for other intrauterine pathologies, and it depended on the surgeon's hysteroscopic experience. Bleeding during or after operation is the second most common complication of hysteroscopy $(0.25 \%$ of all cases), with myomectomy being the procedure with the highest complication rate $(2-3 \%)$ [44]. If bleeding persists postoperatively, a Foley catheter balloon filled with $15-30 \mathrm{ml}$ of fluid can be inserted into the cavity. Pharmacological therapy (e.g., vasopressin and misoprostol), uterine artery embolization, and hysterectomy can also be used if required. Our patient who had uterine bleeding after hysteroscopy responded to $2.5 \mathrm{~g} /$ day of oral conjugated estrogen and was discharged 7 days after hysteroscopy.

Consequently, most patients with RPL have an intrauterine abnormality detected by hysteroscopy, and HSG remains inadequate to diagnose this condition by itself. Diagnostic hysteroscopy should be considered a necessary, successful, and safe investigative procedure for suspected intrauterine pathologies in cases of recurrent spontaneous abortions. We recommend it as a routine procedure instead of HSG. Also, regardless of the results of uterine cavity evaluation, other potential causes of the RPL should be considered thoroughly.

\section{References}

1. Li TC, Iqbal T, Anstie B et al (2002) An analysis of the pattern of pregnancy loss in women with recurrent miscarriage. Fertil Steril 78:1100-1106

2. Knudsen UB, Hansen V, Juul S (1991) Prognosis of a new pregnancy following previous spontaneous abortions. Eur J Obstet Gynecol Reprod Biol 39:31-35

3. Kutteh WH (1998) Recurrent pregnancy loss. In: Car BR, Blackwell RE (eds) Textbook of reproductive medicine, 2nd edn. Appleton and Lange, Stamford, pp 679-692

4. Katz VL, Kuller JA (1994) Recurrent miscarriage. Am J Perinatol 11:386-397

5. Lee RM, Silver RM (2000) Recurrent pregnancy loss: summary and clinical recommendations. Semin Reprod Med 18:433-440

6. Tulppala M, Palosuo T, Ramsay T, Miettien A, Salonen R, Ylikorkala O (1993) A prospective study of 63 couples with a history of recurrent spontaneous abortion: contributing factors and outcome of subsequent pregnancies. Hum Reprod 8:764 770

7. Gilchrist DM, Livingston JE, Hurlburt JA, Wilson RD (1991) Recurrent spontaneous pregnancy loss. Investigation and reproductive follow-up. J Reprod Med 36:184-188

8. Coulam CB (1991) Epidemiology of recurrent spontaneous abortion. Am J Reprod Immunol 26:23-27

9. Bergant AM, Reinstadler K, Moncayo HE, Solder E, Heim K, Ulmer $\mathrm{H}$ et al (1997) Spontaneous abortion and psychosomatics: a prospective study on the impact of psychological factors as a cause for recurrent spontaneous abortion. Hum Reprod 12:1106-1110

10. Christiansen OB, Mathiesen O, Lauritsen JG, Grunnet N (1990) Idiopathic recurrent spontaneous abortion evidence of a familial predisposition. Acta Obstet Gynecol Scand 69:597-601

11. Abbas A, Triphati P, Naik S, Agrawal S (2004) Analysis of human leukocyte antigen (HLA)-G polymorphism in normal women and in women with recurrent spontaneous abortions. Eur J Immunogenet 31:275-278

12. Arslan E, Colakoglu M, Celik C, Gezginc K, Acar A, Capar M, Akoz M, Akyurek C (2004) Serum TNF-alpha, IL-6, lupus anticoagulant and cardiolipin antibody in women with and without a past history of recurrent miscarriage. Arch Gynecol Obstet 270:227-229

13. Tuckerman E, Laird SM, Stewart R, Wells M, Li TC (2004) Markers of endometrial function in women with unexplained recurrent pregnancy loss: a comparison between morphologically normal and retarded endometrium. Hum Reprod 19:196205

14. Stray-Pederson B, Stray-Pederson S (1984) Etiologic factors and subsequent reproductive performance in 195 couples with a prior history of habitual abortion. Am J Obstet Gynecol 148:140-146

15. Tho PT, Byrd JR, McDonough PG (1979) Etiologies and subsequent reproductive performance of 100 couples with recurrent abortion. Fertil Steril 32:389-395

16. Acien P (1996) Uterine anomalies and recurrent miscarriage. Infertil Reprod Med Clin North Am 7:698-719

17. Clifford K, Rai R, Watson H, Regan L (1994) An informative protocol for the investigation of recurrent miscarriage: preliminary experiences of 500 consecutive cases. Hum Reprod 9:1328-1332

18. Raziel A, Kornberg Y, Friedler S et al (2001) Hypercoagulable thrombophilic defects and hyperhomocysteinemia in patients with recurrent pregnancy loss. Am J Reprod Immunol 45:65-71

19. Carp H, Salomon O, Seidman D et al (2002) Prevalence of genetic markers for thrombophilia in recurrent pregnancy loss. Hum Reprod 17:1633-1637

20. Loffer FD (1989) Hysteroscopy with selective endometrial sampling compared with DC for abnormal uterine bleeding: the value of negative hysteroscopic view. Obstet Gynecol $73: 16-20$ 
21. Garuti G, Sambruni I, Cellani D et al (1999) Hysteroscopy and transvaginal ultrasonography in postmenopausal women with uterine bleeding. Int J Obstet Gynecol 65:25-33

22. Marlow JL (1995) Media and delivery systems. Obstet Gynecol Clin North Am 22:409-422

23. Schenk LM, Coddington CC III (1999) Laparoscopy and hysteroscopy. Obstet Gynecol Clin North Am 26:1-22

24. Bacsko G (1997) Uterine surgery by operative hysteroscopy. Eur J Obstet Gynecol Reprod Biol 71:219-222

25. DeCherney AH, Diamond MP, Lavy G (1987) Endometrial ablation for intractable uterine bleeding: hysteroscopic resection. Obstet Gynecol 70:668-670

26. Woelfer B, Salim R, Banerjee S et al (2001) Reproductive outcomes in women with congenital uterine anomalies detected by three-dimensional ultrasound screening. Obstet Gynecol 98:1099-1103

27. Proctor JA, Haney AF (2003) Recurrent first trimester loss is associated with uterine septum but not with bicornuate uterus. Fertil Steril 80:1212-1215

28. Halbrecht I (1951) The role of anatomical changes of the uterine cavity in the pathogenesis of habitual abortion. Gynaecologica 131:1-9

29. Valli E, Zupi E, Marconi D, Vaquero E, Giovannini P, Lazzarin N, Romanini C (2001) Hysteroscopic findings in 344 women with recurrent spontaneous abortion. J Am Assoc Gynecol Laparosc 8:398-401

30. Propst AM, Hill JA III (2000) Anatomic factors associated with recurrent pregnancy loss. Semin Reprod Med 18:341-350

31. March CM, Israel R (1987) Hysteroscopic management of recurrent abortion caused by septate uterus. Am J Obstet Gynecol 156:834-842

32. Fedele L, Dorta M, Brioschi D, Guidici MN, Candiani GB (1989) Pregnancies in septate uteri: outcome in relation to site of uterine implantation as determined by sonography. AJR Am J Roentgenol 152:781-784

33. Dabirashrafi H, Bahadori M, Mohammad K, Alavi M, Moghadami-Tabrizi N, Zandinejad K et al (1995) Septate uterus: new idea on the histologic features of the septum in this abnormal uterus. Am J Obstet Gynecol 172:105-107
34. Valle RF, Sciarra JJ (1988) Intrauterine adhesions: hysteroscopic diagnosis, classification, treatment, and reproductive outcome. Am J Obstet Gynecol 158:1459-1470

35. Valli E, Zupi E, Marconi D, Solima E, Nogar G, Romanini C (1998) Outpatient diagnostic hysteroscopy. J Am Assoc Gynecol Laparosc 5:397-402

36. Barbot J (1995) Hysteroscopy and hysterography. Obstet Gynecol Clin North Am 22:591-603

37. Garuti G, Sambruni I, Colonnelli M, Luerti M (2001) Accuracy of hysteroscopy in predicting histopathology of endometrium in 1500 women. J Am Assoc Gynecol Laparosc 8:207-213

38. Raziel A, Arieli S, Bukovsky I, Caspi E, Golan A (1994) Investigation of the uterine cavity in recurrent aborters. Fertil Steril 62:1080-1082

39. Lindheim SR, Morales AJ (2002) Complications of sonohysterography and hysteroscopy: lessons learned and avoiding pitfalls. J Am Assoc Gynecol Laparosc 9:223-231

40. Jansen FW, Vredevoogd CB, van Ulzen K (2000) Complications of hysteroscopy: a prospective, multicenter study. Obstet Gynecol 96:266-270

41. Wisawasukmongchol W, Bunyavejchevin S, Veeranarapanich S, Suwajananakorn S, Limpaphayom KK (2001) Diagnostic hysteroscopy at King Chulalongkorn Memorial Hospital: 7 years experience. J Med Assoc Thai 84:321-325

42. Loffer FD (1995) Contraindications and complications of hysteroscopy. Obstet Gynecol Clin North Am 22:445-455

43. Castaing N, Darai E, Chuong T, Benifla JL, Bernard G, Meneux E, Tardif D, Batallan A, Madelenat P (1999) Mechanical and metabolic complications of hysteroscopic surgery: report of a retrospective study of 352 procedures. Contracep Fertil Sex 27:210-215

44. Cooper JM, Brady RM (2000) Intraoperative and early postoperative complications of operative hysteroscopy. Obstet Gynecol Clin North Am 27:347-366 